\title{
EDITORIAL
}

\section{Evidence-based practice, a must in medical practice}

'A being of supernatural powers or attributes, believed in and worshiped by a people, especially a male deity thought to control some part of nature or reality', this is one of the definitions of 'God', given in the Oxford English dictionary. This definition may also be taken up by some surgeons, by themselves or by others and on occasion this maybe entirely appropriate. Although this maybe the case we all have a duty of care to the patients we treat and thereby are responsible in providing them with the best possible care available. To do this we must follow an evidence based practice hand in hand with a multidisciplinary approach. Add this to changing times and the availably of vast amounts of freely available information we are also dealing with a new breed of patients who come with pre formed expectations and demands.

Evidence-based medicine has been defined as 'the conscientious, explicit and judicious use of current best evidence in making decisions about the care of individual patient. It has arisen from the critical need of bridging the gap between research and clinical practice, and combines the use of qualitative evidence and applying it to the art of clinical decision making. This form of decision making takes into account the individuality of the patient and their condition, the clinician's expertise and best available research evidence. We have a duty to our patients to provide them with the best possible treatment available and evidence based practice enables us to achieve this goal.

In the past, we have relied greatly on the experience of individual doctors or 'gods' in our profession to guide us to the right decision, although this experience is invaluable it maybe suboptimal on its own in the overall treatment options available especially in the current information era.

So how do we follow evidence based practice? The literature describes five steps. Translation of uncertainty to an answerable question and thereby in the first place asking the question. Then retrieval of the best evidence available, this could be through journals, peer discussion and multidisciplinary approach. Critical appraisal of the information gathered. Application of the results obtained finally evaluation of the performance. The systematic review of published research studies is a major method used for evaluating particular treatments. The Cochrane Collaboration is one of the best-known, respected examples of systematic reviews.

It is also essential that we assess the quality of the evidence available to us and for this a system has been established by the U.S. Preventative services task force, describing levels I to III. Taking into account these systems and evidence clinical guidelines and protocols can be set up to provide uniform care. These too have their good and bad points and should never be considered as rigid structures and here is where the experience of a clinician comes in to provide the best available treatment.

With the establishment of evidence based medicine, a multidisciplinary approach to patient management is an integral part of delivering patient care. Multidisciplinary approach to patient care was first established in the treatment of cancer patients. The main aim was to ensure that patients received timely care from appropriately skilled professionals, that there is continuity of care, and that patients get adequate information and support. Unlike in yester years patient care should be a 'team approach'. Although overall there should be a lead clinician who is responsible. This team should not only treat the patient's condition but also take into account the patients well-being after discharge thereby ensuring a rapid and complication free recovery. The team should include clinicians of primary, secondary and tertiary care as well 
as allied professionals.

Surgery as a profession is changing, for the better. Training methods have changed from being apprenticeships to structured learning but still retaining the traditions of teacher-trainee. Teaching is evidence based and research is encouraged amongst the trainees. A multidisciplinary approach to patient care is encouraged and in some units is becoming the norm. Discussing morbidity and mortality in units provides a platform to discuss complicated cases and pit falls to avoid. This also encourages a transparency and a 'no blame culture' which provides the best care for the patient. The current problems of not accepting responsibility and asking for help even as consultants when faced with a challenging case does not provide the best outcome for the patients. Patient care is a privilege bestowed upon us, to abuse this is ethically wrong, therefore we must take all available measures to provide the best possible care available, taking into account the resources available to us.

\section{Ruvini Abeygunaratne}

